\title{
High Perioperative Inspiratory Oxygen Fraction to Prevent Anastomotic Dehiscence
}

\author{
Christian Sahlholt Meyhoff • Jørn Wetterslev • \\ Lars Nannestad Jorgensen • Lars Simon Rasmussen
}

Received: 15 March 2012 / Accepted: 12 November 2012 /Published online: 27 November 2012

(C) 2012 The Society for Surgery of the Alimentary Tract

Dear editor,

We read with interest the article by Schietroma et al., ${ }^{1}$ in which complications from anastomotic dehiscence are added to the current debate regarding possible beneficial effects of a high perioperative inspiratory oxygen concentration.

The primary outcome, anastomotic dehiscence, is highly clinically relevant, but we are concerned that statistical power may not have been adequate in this trial. It is not described which difference the authors were trying to detect between the two groups and we cannot see why 72 patients were considered to constitute a reasonable sample. The authors found anastomotic dehiscence in 4 out of 35 patients vs. 8 out of 37 patients given 80 and $30 \%$ oxygen, respec-

\section{S. Meyhoff $(\square)$}

Department of Anaesthesiology, Copenhagen University Hospital, Herlev, Herlev Ringvej 75,

DK-2730 Herlev, Denmark

e-mail: christianmeyhoff@gmail.com

J. Wetterslev

Copenhagen Trial Unit, Centre for Clinical Intervention Research, Department 3344, Rigshospitalet, Copenhagen University

Hospital, Copenhagen, Denmark

L. N. Jorgensen

Department of Surgery K, Bispebjerg Hospital, University

of Copenhagen, Copenhagen, Denmark

L. S. Rasmussen

Department of Anaesthesia, Centre of Head and Orthopaedics, Copenhagen University Hospital, Rigshospitalet, Copenhagen, Denmark tively, and mention that this was significantly different $(p<0.05)$. Using a two-sided Fisher's exact test, however, the $p$ value is 0.35 , and the corresponding relative risk and confidence interval are not correct either.

Wound infection occurred in 5 out of 35 patients vs. 10 out of 37 patients, and that is also not significantly different $(p=0.25)$.

The findings by Schietroma et al. are interesting, but not very conclusive, and there is still a major gap of knowledge before $80 \%$ oxygen may be considered for inclusion in surgical care improvement projects to reduce the rates of surgical site infections and anastomotic dehiscence.

Conflict of Interest All authors of this letter were members of the PROXI trial steering committee investigating $80 \%$ oxygen to prevent surgical site infection (JAMA 2009;302:1543-50) and are also authors of an upcoming Cochrane review about the effects of high perioperative inspiratory oxygen fraction for adult surgical patients (Cochrane Database of Systematic Reviews 2010, Issue 12. Art. No.: CD008884. DOI:10.1002/14651858.CD008884)

\section{Reference}

1. Schietroma M, Carlei F, Cecilia EM, Piccione F, Bianchi Z, Amicucci G. Colorectal infraperitoneal anastomosis: the effects of perioperative supplemental oxygen administration on the anastomotic dehiscence. J Gastrointest Surg 2012; 16: 427-434. 